\title{
Quercetin inhibits the growth of human gastric cancer stem cells by inducing mitochondrial-dependent apoptosis through the inhibition of PI3K/Akt signaling
}

\author{
XINSHENG SHEN $^{1}$, YAQING SI ${ }^{1}, \mathrm{ZHAO} \mathrm{WANG}^{1}$, JIACHEN WANG ${ }^{1}$, YONGQIANG GUO $^{1}$ and XIEFU ZHANG ${ }^{2}$ \\ Departments of ${ }^{1}$ Minimally Invasive Surgery and ${ }^{2}$ Gastrointestinal Surgery, \\ The First Affiliated Hospital of Zhengzhou University, Zhengzhou, Henan 450052, P.R. China
}

Received June 8, 2015; Accepted May 4, 2016

DOI: $10.3892 / \mathrm{ijmm} .2016 .2625$

\begin{abstract}
Cancer stem cells (CSCs) have recently been linked to new treatment strategies for gastric cancer due to the critical role which they play as the 'heartbeat' of cancer. In the present study, we explored the effects of quercetin, an anti-inflammatory and antiviral compound, on gastric CSCs (GCSCs). We noted that quercetin exerted pronounced inhibitory effects on GCSC survival. Moreover, quercetin induced cell apoptosis in a mitochondrial-dependent manner, as shown by the reduction in mitochondrial membrane potential, the activation of caspase- 3 and -9 , and the downregulation of $\mathrm{Bcl}-2$, as well as the upregulation of Bax and cytochrome $c$ (Cyt-c). Additionally, a marked decrease in Akt phosphorylation levels was observed following treatment with quercetin, whereas pre-treatment with fumonisin B1 (FB1, Akt activator) significantly attenuated the inhibitory effects of quercetin on cell growth and its promoting effects on mitochondrial-dependent apoptosis. Notably, FB1 enhanced the expression of $\mathrm{Bcl}-2$, which was inhibited by quercetin, and prevented the decrease in mitochondrial membrane potential induced by quercetin. However, the increase in the levels of caspases, Bax and Cyt- $c$ induced by quercetin was also attenuated by the addition of FB1 to the GCSCs. Therefore, our results demonstrate that quercetin triggers mitochondrial apoptotic-dependent growth inhibition via the blockade of phosphoinositide 3-kinase (PI3K)-Akt signaling in GCSCs, indicating a potential target for the treatment of gastric cancer.
\end{abstract}

Correspondence to: Dr Xinsheng Shen, Department of Minimally Invasive Surgery, The First Affiliated Hospital of Zhengzhou University, Zhengzhou, Henan 450052, P.R. China

E-mail: xinshengshen@163.com

Dr Xiefu Zhang, Department of Gastrointestinal Surgery, The First Affiliated Hospital of Zhengzhou University, 1 East Jianshe Road, Zhengzhou, Henan 450052, P.R. China

E-mail: xiefu_zhang@126.com

Key words: quercetin, gastric cancer stem cells, cell growth, mitochondria, phosphoinositide 3-kinase/Akt

\section{Introduction}

Despite a recent decline in incidence, gastric cancer (GC) remains one of the most common health issues with a significant mortality rate $(1,2)$. There are approximately 800,000 GC-related deaths worldwide each year $(1,3)$. However, the pathogenetic mechanisms and the origin of GC are not yet clear. Previous research has postulated that, by contrast to conventional stochastic theory, cancers may initiate from a rare subpopulation of cells within the tumor, known as cancer stem cells (CSCs), which have been considered the 'heartbeat' of cancer $(4,5)$. This theory can be traced back to 1997, when Bonnet and Dick first isolated stem cells from acute myeloid leukemia (AML) (6). Since then, CSCs have been discovered in various types of tumors, including breast (7), prostate (8), liver (9), colon (10) and pancreatic (11) cancer. Gastric CSCs (GCSCs) have already been isolated, identified and proven to drive the growth and metastasis of GC cells $(12,13)$. Moreover, GCSCs possess chemo-resistant and radio-resistant abilities and differ from their parental cancer cells $(14,15)$. The emergence of GCSCs may lead to a paradigm shift in GC research, and may assist in the establishment of a novel therapeutic tool for the treatment of GC.

Quercetin (3,3',4',5,7-pentahydroxyavone), which is widely found in edible fruits and vegetables, exerts anti-inflammatory and antiviral effects (16). Increasing attention has been paid to its anti-tumor effects on many cancer cell models, including GC (17,33). Quercetin inhibits the development of GC possibly by regulating cell cycle arrest or by inducing protective autophagy (18-20). Studies on the synergetic, inhibitory effects of quercetin on the self-renewal ability of pancreatic CSCs have noted the potential selective toxicity of quercetin to pancreatic CSCs (21-23). Moreover, this synergetic phenomenon was also observed in prostate CSCs by Tang et al (24). Moreover, Chang et al showed that quercetin significantly reduced self-renewal, stemness signature expression and the migratory ability of head and neck cancer-derived sphere cells (25). However, to the best of our knowledge, no studies regarding the effects of quercetin on GCSCs were completed prior to the present study. Thus, in this study, we explored the role of quercetin in controlling the proliferation and apoptosis of GCSCs, and aimed to elucidate the potential molecular mechanisms involved. 


\section{Materials and methods}

Culture of parental and spheroid body-forming cells. The human GC cell line, MGC803, was obtained from the Cell Bank of the Chinese Academy of Sciences (Shanghai, China). The cells were cultured in RPMI-1640 medium (Gibco-BRL, Grand Island, NY, USA) containing 10\% fetal bovine serum (FBS) (Gibco, Rockville, MD, USA), $100 \mathrm{kU} / 1$ penicillin, $0.1 \mathrm{~g} / 1$ streptomycin, $0.3 \mathrm{~g} / \mathrm{l} \mathrm{L-glutamine}$ (Invitrogen, Carlsbad, CA, USA) and $0.85 \mathrm{~g} / 1$ $\mathrm{NaHCO}_{3}$ at $37^{\circ} \mathrm{C}$ in a humidified atmosphere containing $5 \% \mathrm{CO}_{2}$. Spheroid bodies were derived from parental cells. Briefly, the parental cells were first cultured in serum-free RPMI-1640 medium with $20 \mathrm{ng} / \mathrm{ml}$ human fibroblast growth factor 2 (FGF2), $100 \mathrm{ng} / \mathrm{ml}$ epidermal growth factor (EGF) (Chemicon, Japan), 1\% N-2 supplement, 2\% B-27 supplement (Invitrogen) and $1 \%$ antibiotic mixture (Gibco). First, parental GC cells were plated into 96-well ultra-low attachment plates at 1,000 cells/well. Subsequently, the formation of spheroid bodies was observed under an inverted microscope once in a while. Primary spheroid bodies were noted within 2 weeks. The spheroid bodies were then collected and dissociated by trypsinization for passage and amplification in serum-free medium.

Colony formation assay. The subspheroid body formation assay was performed using crystal violet staining. The collected spheroid bodies were dissociated and centrifuged into single suspended cells. The cells were seeded into 6-well plates at a density of $1 \times 10^{2}$ cells/well, as well as the digested GC cells. The serum-free culture medium was changed every 3-4 days. Two weeks later, the culture medium was removed and the cells were washed in PBS 3 times. Subsequently, $4 \%$ paraformaldehyde was added for $30 \mathrm{~min}$ to fix the cells, which were then stained with crystal violet (Sigma, St. Louis, MO, USA) for $10 \mathrm{~min}$. Obvious cell colonies were observed under an inverted microscope following 10 washes with PBS. Colonies containing $<50$ cells were excluded.

Reverse transcription-quantitative polymerase chain reaction $(R T-q P C R)$. A Qiagen RNeasy mini kit (Qiagen, Valencia, CA, USA) was used for total RNA extraction from the parental cells and spheroid body-forming cells. Agarose gel electrophoresis was used to detect the integrity and purification of RNA samples. Absorption under $260 \mathrm{~nm}$ was used to determine the RNA concentration. cDNA synthesis was conducted using a High-Capacity cDNA Reverse Transcription kit (Applied Biosystems, Foster City, CA, USA); qPCR was performed with SYBR-Green (Takara, Dalian, China) as a fluorescent dye to determine the changes in the levels of stem-specific genes [octamer-binding transcription factor 4 (Oct4), SRY (sex determining region Y)-box 2 (Sox2), Nanog and CD44] following the manufacturer's instructions. $\beta$-actin was used as a normalizing control. The primer sequences were as follows: Oct4, 5'-AGCCCTCATTTCACCAGGCC-3' (forward) and 5'-CCC CCACAGAACTCATACGG-3' (reverse); Sox 2, 5'-GAAAAA CGAGGGAAATGGG-3' (forward) and 5'-GCTGTCA TTTGCTGTGGGT-3' (reverse); Nanog, 5'-CTCCTCCCA TCCCTCATA-3' (forward) and 5'-AGGCTCCAACCATA CTCC-3' (reverse); CD44, 5'-CATCCCAGACGAAGACA GTCC-3' (forward) and 5'-TGATCAGCCATTCTGGAA TTTG-3' (reverse); and $\beta$-actin, 5'-CTGAGAGGGAAATCGT
GCGT-3' (forward) and 5'-CCACAGGATTCCATACCC AAGA-3' (reverse).

MTT assay. An in vitro analysis of the cytotoxic effects of quercetin on GCSCs was carried out by MTT assay. In brief, $200 \mu \mathrm{l}$ cells were seeded into a 96-well plate and incubated for $12 \mathrm{~h}$, and the medium was then removed. Following treatment with various concentrations of quercetin $(0,20,40,60,80$ and $100 \mu \mathrm{M})$ for the indicated perdios of time at $37^{\circ} \mathrm{C}, 20 \mu 15 \mathrm{mg} /$ ml MTT (Sigma) solution were added to each well. Following a $4 \mathrm{~h}$ of incubation, DMSO $(100 \mu \mathrm{l})$ was added to dissolve the formazan crystals for $15 \mathrm{~min}$. The optical density at $570 \mathrm{~nm}$ was measured to calculate the inhibitory function of quercetin from the cytotoxicity curves. Cells without treatment were used as controls. Quercetin (99\% pure) was purchased from Sigma, dissolved in DMSO, and stored at $-20^{\circ} \mathrm{C}$. Its structure is illustrated in Fig. 1A. The half maximal inhibitory concentration $\left(\mathrm{IC}_{50}\right)$ was defined as the drug concentration which inhibited cell growth by $50 \%$, and was calculated using a linear regression based on the results of MTT assay.

Cell sorting by flow cytometry. The cells were counted and transferred to a 5-ml tube, washed with HBSS containing $2 \%$ heat-inactivated FBS, and we reconstituted cells at a concentration of $10^{6} / 100 \mu \mathrm{l}$ with Sandoglobulin solution (Sandoz Pharmaceuticals Corp., East Hanover, NJ, USA). Subsequently, the cells were incubated on ice for $20 \mathrm{~min}$. After washing twice with HBSS, the cells were resuspended in HBSS containing $1 \mathrm{mM}$ HEPES and $2 \%$ FBS. After filtering with a sterile mesh filter $(40 \mu \mathrm{m})$, the cells were incubated with anti-CD44-FITC at a dilution of 1:400 (no. 560977; BD Biosciences, Mountain View, CA, USA) for $30 \mathrm{~min}$. The cells were then rinsed with HBSS and subsequently resuspended in HBSS solution with $2 \%$ FBS, $1 \mathrm{mM}$ HEPES and $1 \%$ penicillin-streptomycin sulfate. Subsequently, the samples were examined using FACSAria (serial no. 342975; BD Immunocytometry Systems, Franklin Lakes, NJ, USA) for fluorescence-activated cell sorting. The cells were routinely sorted twice, as previously described (26).

Determination of apoptosis by flow cytometry. The quantification of the apoptosis of the GCSCs treated with quercetin was examined by flow cytometry. The cells were treated with $53.0 \mu \mathrm{M}$ quercetin for $24 \mathrm{~h}$, and then centrifuged at $1,000 \mathrm{x} \mathrm{g}$ for 5 min, and washed twice with PBS. An Annexin V-fluorescein isothiocyanate (FITC) apoptosis detection kit (Invitrogen) was used for the detection of apoptotic cells. After staining with $5 \mu \mathrm{l}$ Annexin V-FITC and $5 \mu \mathrm{l}$ propidium iodide for $15 \mathrm{~min}$ in the dark at room temperature, the cells were immediately subjected to flow cytometric observation and analysis within $1 \mathrm{~h}$.

Determination of mitochondrial membrane potential. The resultant fluorescence intensity was used as an arbitrary unit representing mitochondrial membrane potential. The GCSCs were treated with the AKT activator, fumonisin B1 (FB1, $25 \mu \mathrm{M}$ ) (Sigma) for $2 \mathrm{~h}$, in prior to exposure to quercetin for $24 \mathrm{~h}$ and rinsed with PBS. The cells were resuspended in $1 \mathrm{ml}$ rhodamine $123\left(1 \mu \mathrm{g} / \mathrm{ml}\right.$; Sigma) for $30 \mathrm{~min}$ in the dark at $37^{\circ} \mathrm{C}$. After rinsing with PBS 3 times, the fluorescence intensity of the cells was examined by fluorescence-activated cell sorting at $507 \mathrm{~nm}$ (excitation) and $529 \mathrm{~nm}$ (emission). 
A<smiles></smiles>

Quercetin
B

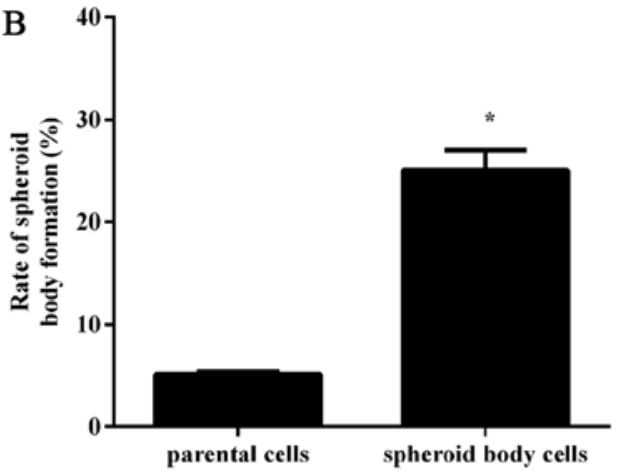

D

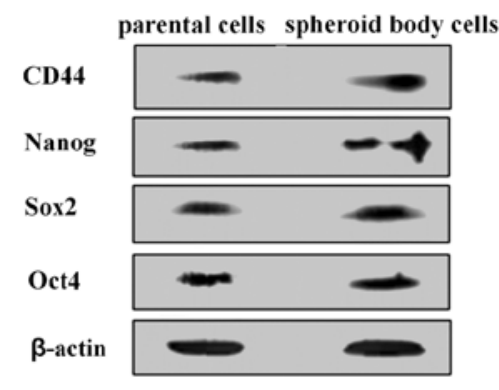

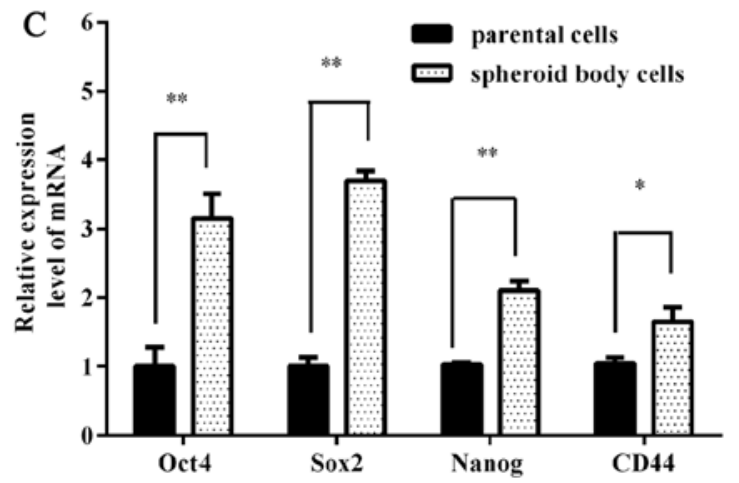

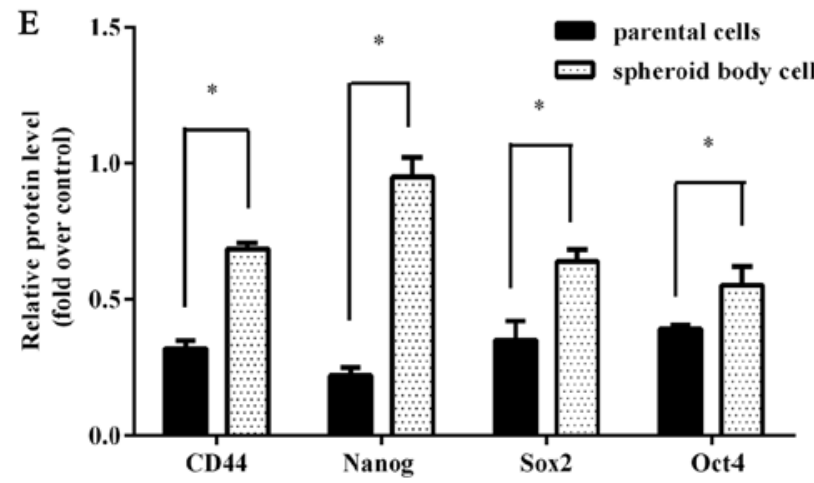

Figure 1. Isolation and identification of spheroid bodies from MGC803 cells. (A) The structure of quercetin. (B) Two weeks later, these spheroid body-forming cells generated subspheroid bodies, and the subspheroid body formation rate was compared with that of the parental cells through crystal violet staining. (C) RT-qPCR illustrated the relative mRNA expression of octamer-binding transcription factor 4 (Oct4), SRY (sex determining region Y)-box 2 (Sox2), Nanog and CD44 in the spheroid body-forming cells compared with the parental cells. (D) Western blot analysis of the level of gastric cancer stem cell (GCSC)-related proteins (CD44, Nanog, Sox 2 and Oct4). (E) The relative protein levels were also determined. ${ }^{*}$ p $<0.05$ vs. parental group; ${ }^{* *}$ p $<0.01$ vs. parental group.

Caspase activity assay. Caspase activity was measured using a caspase colorimetric assay kit from Sigma. Briefly, the cells were treated with quercetin for $24 \mathrm{~h}$ and then lysed. The centrifugal supernatant was placed into a 96-well microplate with reaction buffer. The microplate was incubated at $37^{\circ} \mathrm{C}$ overnight and substrate was added. The activity was measured at $405 \mathrm{~nm}$ using a spectrophotometer (U-5100; Techcomp Ltd., Shanghai, China).

Western blot analysis. Before western blot analysis was performed, the cells were lysed with RIPA lysis buffer [100 mM $\mathrm{NaCl}, 50 \mathrm{mM}$ Tris-HCl pH 7.5, $1 \%$ Triton X-100, 1 mM ethylenediaminetetraacetic acid (EDTA), $10 \mathrm{mM} \beta$-glycerophosphate and $2 \mathrm{mM}$ sodium vanadate and protease inhibitor] to collect whole-cell extracts. To prepare the cytosolic extracts, the cells were lysed with RIPA lysis buffer containing protease inhibitor cocktail for $30 \mathrm{~min}$ on ice, and were then homogenized by 6 passages through a 25 -gauge needle. Following centrifugation $(14,000 \mathrm{x} \mathrm{g})$ at $4^{\circ} \mathrm{C}$ for $10 \mathrm{~min}$, the resultant supernatant (cytosol) was subjected to western blot analysis to evaluate the release of cytochrome $c$ (Cyt-c) . The protein samples were then separated using SDS-PAGE on a $12 \%$ gel and transferred onto polyvinylidene difluoride membranes (Amersham Pharmacia, Freiburg, Germany). Non-specific binding was blocked by incubation with 5\% non-fat milk in Tris-buffered saline with Tween-20 (TBST) buffer at room temperature for $1 \mathrm{~h}$. Incubation with the primary antibody was carried out overnight in a cold room, and the membranes were subsequently washed in TBST 3 times. Primary antibodies were as follows: anti-Bax (no. 2774,
1:400), anti-Bcl-2 (no. 2872, 1:200), anti-Cyt-c (no. 4272, $1: 300$ ) and anti- $\beta$-actin (no. 4967S) were purchased from Cell Signaling Technology (Danvers, MA, USA). A secondary antibody conjugated to horseradish peroxidase (1:5,000; Sigma) was added to the membranes followed by incubation for $1 \mathrm{~h}$ at room temperature, followed by enhanced chemiluminescence using an ECL kit (Amersham Pharmacia, Piscataway, NJ, USA). Finally, protein bands were visualized using ECL and quantified using Quantity One software (Bio-Rad, Hercules, CA, USA).

Statistical analysis. All experimental data were derived from at least 3 independent experiments, and all values are expressed as the means \pm SD. Statistical analyses were performed using the SPSS 16.0 statistical software package (SPSS Inc., Chicago, IL, USA). Any differences were assessed using the Student's $\mathrm{t}$-test or one-way ANOVA. A value of $\mathrm{p}<0.05$ was considered to indicate a statistically significant difference.

\section{Results}

Isolation of GCSCs from MGC803 cells. The MGC803 parental cells cultured in serum-free medium grew as non-adherent, three-dimensional spheroid clusters, termed spheroid bodies. As time progressed, the cell spheroid bodies gradually increased not only in volume, but also in number. The self-renewal capacity of these spheroid body-forming cells was preliminarily assessed by counting the subspheroid bodies that they generated. As shown in Fig. 1B, compared 

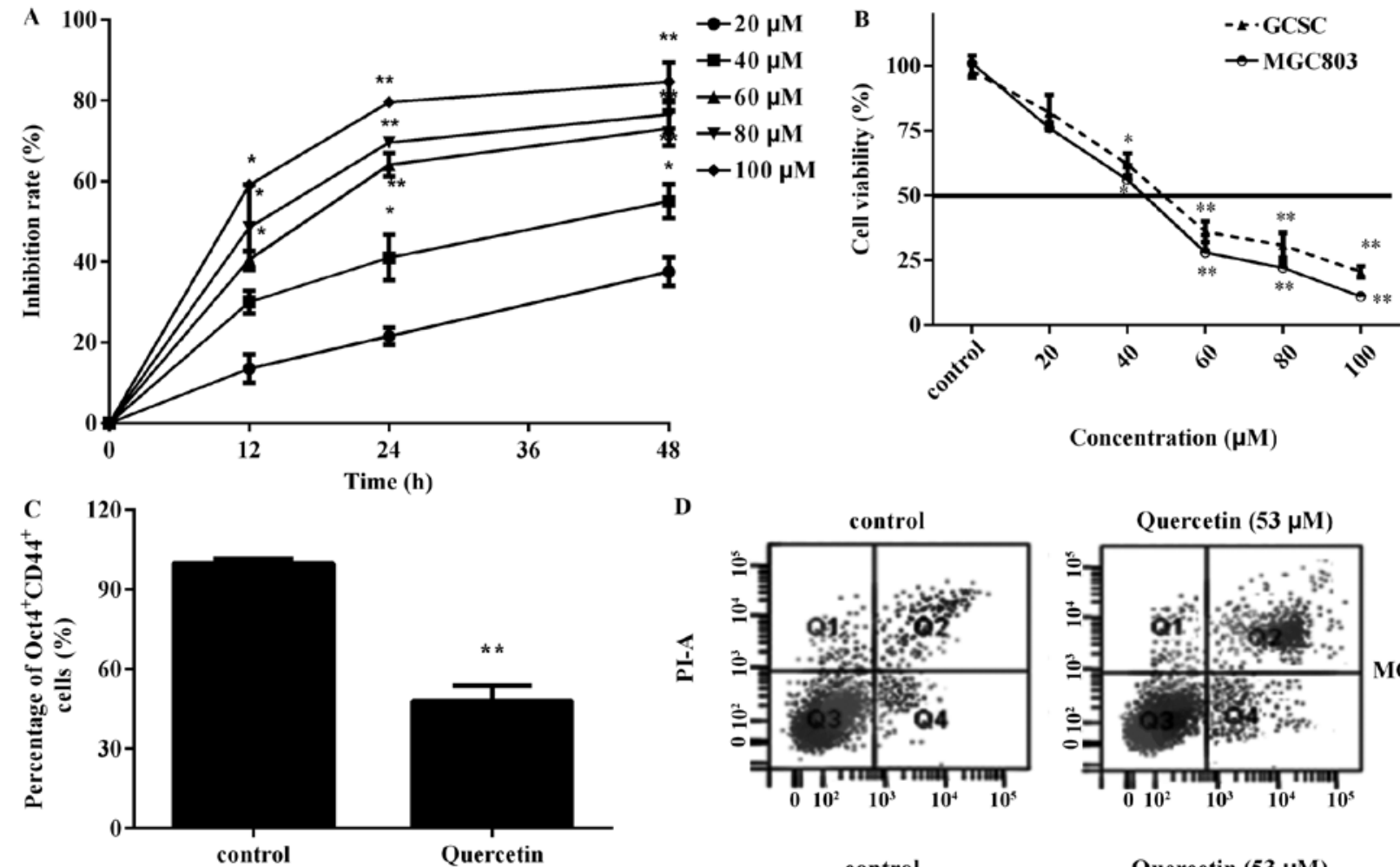

D

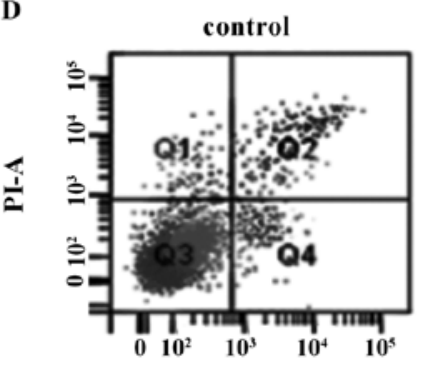

Concentration $(\mu \mathrm{M})$
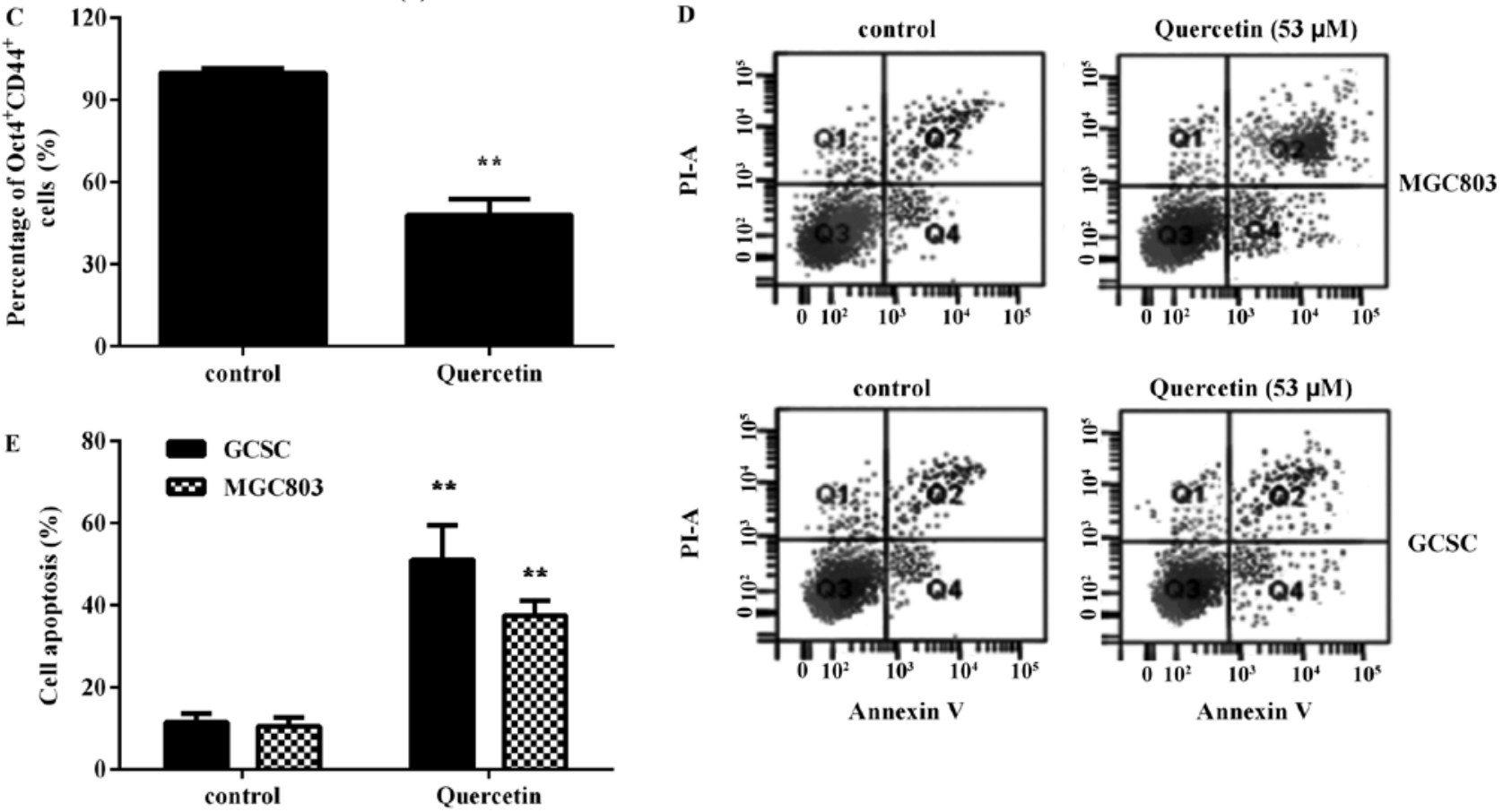

exin $\mathbf{V}$

Figure 2. Effects of quercetin on the growth of gastric cancer stem cells (GCSCs). (A) Increasing concentrations of quercetin (20, 40, 60, 80 and $100 \mu \mathrm{M})$ were added and the cells were incubated for different periods of time (12, 24,36 and $48 \mathrm{~h}$ ), and the absorption at $570 \mathrm{~nm}$ was measured. (B) After $24 \mathrm{~h}$ of treatment, the dose-dependent effects of quercetin $(20,40,60,80$ and $100 \mu \mathrm{M})$ on cell viability were determined by MTT assay. (C) Effects of quercetin on GCSCs. Cells were treated with $53 \mu \mathrm{M}\left(\mathrm{IC}_{50}\right.$ value) quercetin for $24 \mathrm{~h}$. Flow cytometry was applied to detect the percentage of cells bearing the stemness markers, Oct4 and CD44 $\left(\mathrm{Oct} 4^{+} \mathrm{CD} 44^{+}\right.$cells). (D) Flow cytometry was applied for the detection of quercetin-induced cell apoptosis after $24 \mathrm{~h}$ of treatment with quercetin at $53 \mu \mathrm{M}$. (E) The quantification of cell apoptosis is also demonstratedted by the values in the quadrants Q4 (early apoptotic cells) and Q2 (late apoptotic cells) shown in (E). ${ }^{*} \mathrm{p}<0.05$ and ${ }^{* *} \mathrm{p}<0.01$ compared to control groups.

with the parental cells, the rate of spheroid body formation was $25.4 \pm 3.51 \%$ in the spheroid body cells, whereas for the parental cells it was only $5.0 \pm 1.04 \%$, reflecting the high capacity for self-renewal of these isolated spheroid body cells. Furthermore, the results of RT-qPCR revealed that the mRNA levels of stem-specific Oct4, Sox2, Nanog and CD44 in the spheroid body-forming cells were significantly higher than those in the parental cells (Fig. 1C). In addition, the results of western blot analysis revealed the high expression of the stemcell markers, Oct4, Sox 2, Nanog and CD44, in the spheroid body cells, as compared to the parental cells (Fig. 1D). The relative protein levels of CD44, Nanog, Sox 2 and Oct4 in the spheroid body-forming cells were approximately double those of the parental cells, at least for CD44, Nanog and Sox2 (Fig. 1E). Taken together, these results indicate the successful isolation of GCSCs from the MGC803 GC cells.
Quercetin inhibits the viability of GCSCs. To determine the cytotoxity of quercetin on the GCSCs, we used an MTT assay to examine cell viability. As shown in Fig. 2A, treatment with quercetin significantly increased the inhibition rate of viable cells in a time- and dose-dependent manner. Moreover, after treating the GCSC cells with 20 and $100 \mu \mathrm{M}$ quercetin for $24 \mathrm{~h}$, cell viability decreased to $82 \pm 6.73 \%(20 \mu \mathrm{M})$ and $20.5 \pm 2.10 \%(100 \mu \mathrm{M})$ (Fig. 2B), and quercetin strongly restrained GCSC cell viability with an $\mathrm{IC}_{50}$ of approximately $53 \mu \mathrm{M}$. Additionally, we noted that the MGC803 cells were more sensitive than the GCSCs to quercetin, reflecting the relative stronger chemoresistance of GCSCs in comparison to the parental cells. Moreover, a decrease of $>50 \%$ in the number of Oct $4^{+} \mathrm{CD} 44^{+}$cells was observed following treatment with quercetin (Fig. 2C), indicating the diminished proliferative ability of GCSCs upon treatment with quercetin. 
A

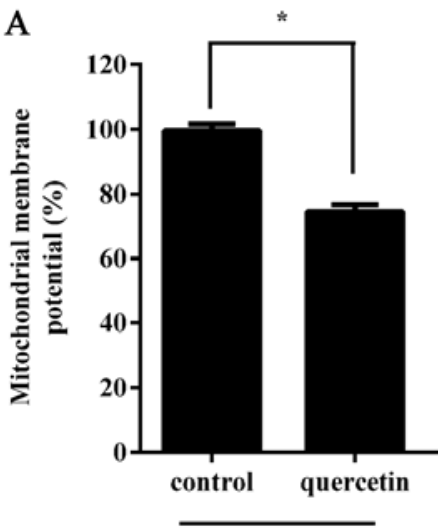

$12 \mathrm{~h}$

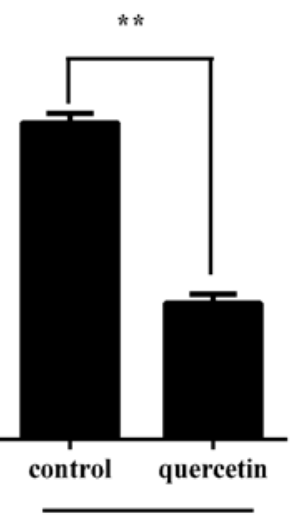

$24 \mathrm{~h}$

C

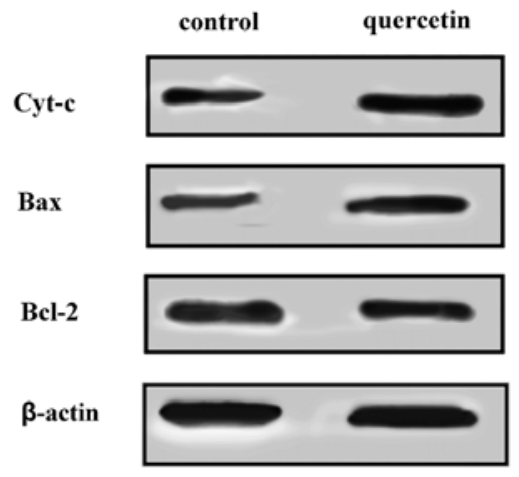

B

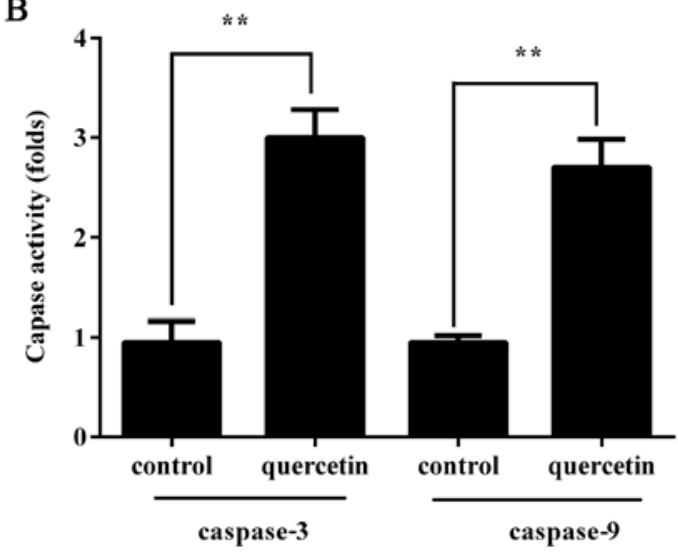

D

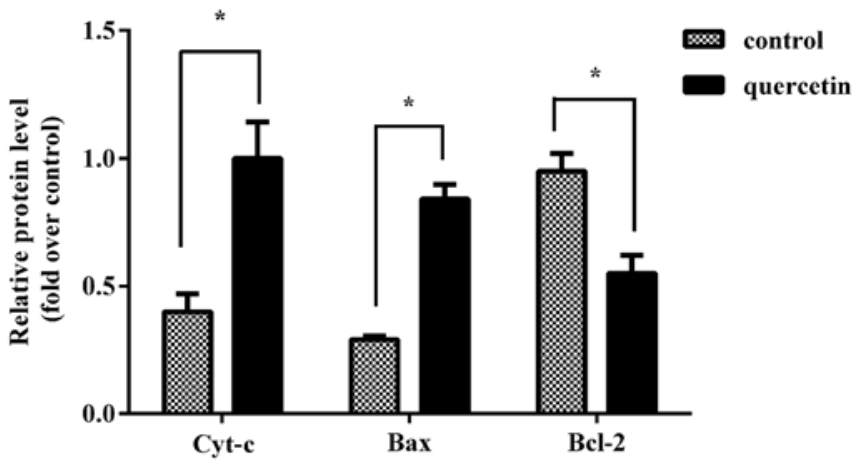

Figure 3. Effects of quercetin on mitochondrial-related apoptosis. (A) Cells were trypsinized and then stained with rhodamine 123 (10 $\mu \mathrm{M})$ for 10 min. A fluorescence spectrophotometer was used to analyze the fluorescence intensity of the cells at $480 \mathrm{~nm}$ (excitation) and $530 \mathrm{~nm}$ (emission). (B) Following treatment wiht quercetin $(53 \mu \mathrm{M})$ for $24 \mathrm{~h}$, caspase-3 and caspase-9 activities were detected by a colorimetric assay. (C) Western blot analysis of mitochondrial apoptotic pathway proteins in the control and quercetin-treated groups. (D) The quantification of Bcl-2, Bax and cytochrome $c$ (Cyt-c) was also determined. ${ }^{*} \mathrm{p}<0.05$ and ${ }^{* * *} \mathrm{p}<0.01$.

Quercetin induces the apoptosis of GCSCs. In order to further analyze the effects of quercetin on GCSC survival, flow cytometry was used to assess quercetin-induced apoptosis. As shown in Fig. 2D, in comparison with the control (untreated) group, quercetin significantly induced apoptosis. When the cells were treated with $53.0 \mu \mathrm{M}$ quercetin for $24 \mathrm{~h}$, GCSC apoptosis was significantly enhanced (Fig. 2E). However, the sensitivity of the GCSCs to quercetin was less than that of the MGC803 cells. These data reflect the inhibitory effects which quercetin exerts on the growth of GCSCs; however, the mechanisms involved require further investigation.

Quercetin and the mitochondrial apoptotic pathway. Mitochondrial apoptosis plays an important role in GC growth inhibition. To ascertain the underlying mechanisms involved in the quercetin-triggered inhibition of cell growth, the mitochondrial apoptotic pathway was analyzed. As shown in Fig. 3A, quercetin decreased mitochondrial membrane potential in a time-dependent manner. The fluorescence intensity at $12 \mathrm{~h}$ was higher than after $24 \mathrm{~h}$ of treatment with quercetin. Concomitantly, as central molecules of the apoptotic cascade, caspase-3 and caspase- 9 were also investigated, and we noted that quercetin upregulated the expression levels of both caspase-3 and caspase-9 (Fig. 3B). Our findings demonstrated that quercetin-induced apoptotic cell death was associated with the activation of caspase-3 and caspase-9. In order to further explore the involvement of the mitochondrial apoptotic pathway in the quercetin-induced inhibition of GCSC growth, the expression of Bax, Bcl-2 and Cyt-c was examined by western blot anlaysis. As shown in Fig. 3C, an increase in the levels of Bax and Cyt-c occurred, together with a significant decrease in the expression of the anti-apoptotic protein, $\mathrm{Bcl}-2$, upon treatment with quercetin. The variations in the levels of these proteins can be directly noted in Fig. 3D. These results suggest that quercetin inhibits the growth of GCSCs by regulating the mitochondrial apoptotic pathway.

Akt is involved in quercetin-induced growth inhibition. Previous studies have demonstrated that quercetin induces cell apoptosis in many types of cancer by blocking the phosphoinositide 3-kinase (PI3K)-Akt pathway, which plays a negative role in the intrinsic mitochondrial pathway (27-29). Therefore, in the present study, the phosphorylation of Akt was investigated. As was expected, an attenuated level of p-Akt was observed following treatment with quercetin (Fig. 4A). Treatment with quercetin induced only a slight decrease in the expression of Akt (Fig. 4B). On the other hand, the addition of fumonisin B1 (FB1, Akt activator) significantly attenuated the inhibitory effects of quercetin on Akt phosphorylation (Fig. 4A). Moreover, the quercetin-induced inhibition of GCSC viability was almost completely abrogated once Akt was activated by treatment with FB1 (Fig. 4C). At the same time, the upregulation of cell apoptosis induced by quercetin was also markedly decreased when the cells were pre-treated with FB1 (Fig. 4D). 
A

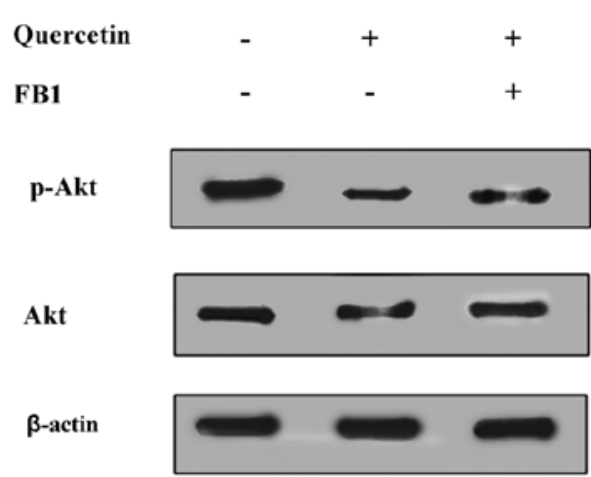

$\mathrm{C}$

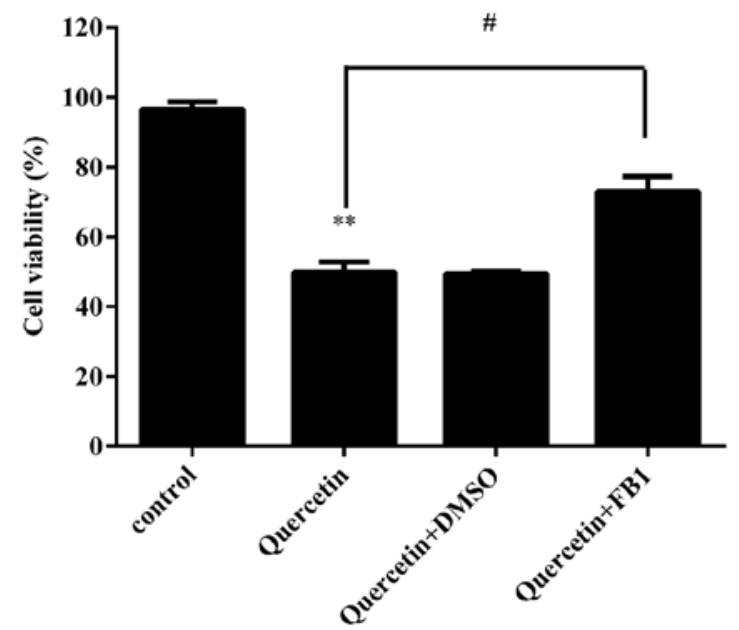

B

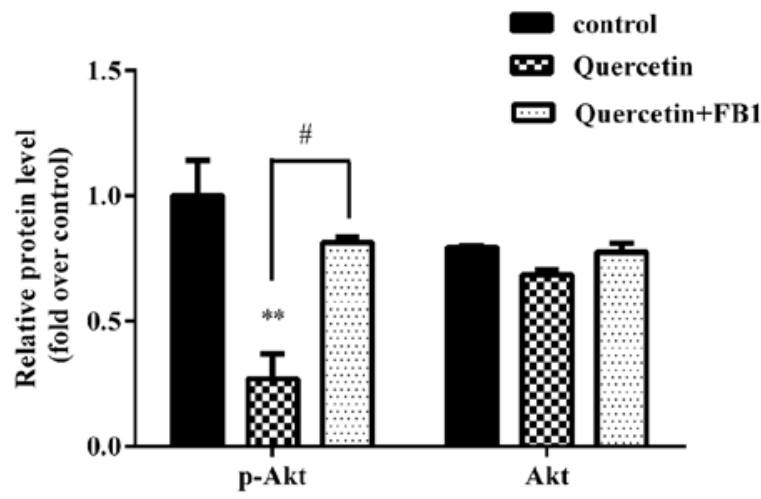

D

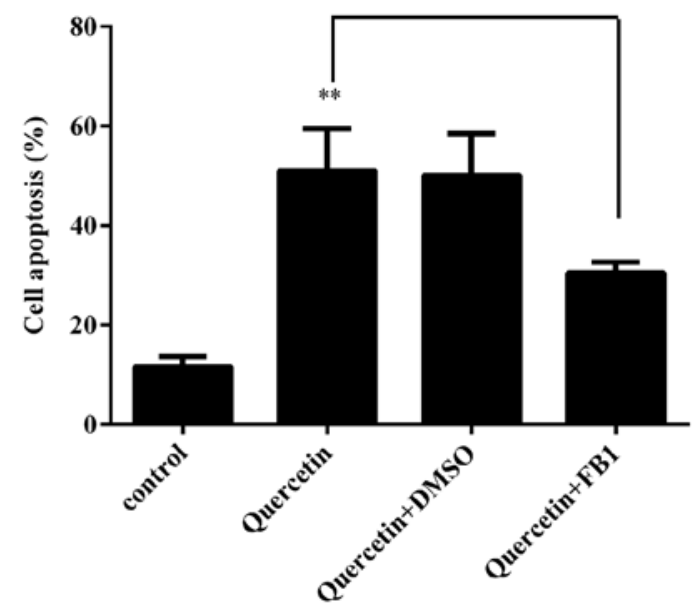

Figure 4. Effects of quercetin on Akt expression. Cells were treated with quercetin (53 $\mu \mathrm{M})$ for $24 \mathrm{~h}$, and the level of Akt and p-Akt was detected by (A) western blot analysis, (B) and these results were then quantified. (C) When gastric cancer stem cells (GCSCs) were pre-treated with the Akt activator, fumonisin B1 (FB1, $25 \mu \mathrm{M}$ ) for $2 \mathrm{~h}$, the protective effects of FB1 on $53 \mu \mathrm{M}$ quercetin-induced cytotoxicity were detected by MTT assay. (D) Quercetin-induced cell apoptosis was also measured by means of flow cytometry. ${ }^{* *} \mathrm{p}<0.01$ and $^{\#} \mathrm{p}<0.05$.

These results suggest that the PI3K-Akt signaling pathway is involved in the quercetin-induced inhibition of the growth of GCSCs.

Association between PI3K-Akt and the mitochondrial pathway. In order to gain further insight into the involvement of Akt in the mitochondrial apoptotic pathway, we examined the influence of FB1 on the quercetin-induced mitochondrial pathway. As shown in Fig. 5A, treatment with quercetin significantly reduced mitochondrial membrane potential, while the addition of FB1 reversed this effect. FB1 markedly abated the increase in the level of caspase-3 (Fig. 5B) and caspase-9 (Fig. 5C) induced by quercetin. Furthermore, the effects of quercetin on the expression of Cyt-c, Bax and $\mathrm{Bcl}-2$ were attenuated by the Akt activator, FB1 (Fig. 5D). Their relative levels were calculated and are depicted in Fig. 5E. These results clearly illustrate the involvement of Akt in the quercetin-induced activation of the mitochondrial apoptotic pathway.

\section{Discussion}

The incidence of GC, which is increasingly perceived as a leading cause of mortality particularly in developing countries, has increased due to the increased aging populations.
Conventional curative treatments, such as surgery, chemotherapy and radiation therapy, are not completely effective, and thus GCSC theory provides new insight into the study of tumor initiation, as well as the development of novel strategies for GC treatment $(30,31)$. Recently, modern tools have been used to investigate the characteristics of GCSCs, including self-renewal pathways, chemoresistance mechanisms and even microRNA expression profiles $(31,32)$. In spite of the significance of GCSCs for GC genesis and development, limited research has been undertaken on the control of GCSCs by effective compounds which serve to block the origination of GCs.

Quercetin (3,3',4',5,7-pentahydroxyavone) is a plant-based chemical that is commonly found in various types of fruits and vegetables (33). Although quercetin has been evaluated in relation to several types of cancer $(17,22,24,33)$, very little is known about its effects on GCSCs. In the present study, we provide evidence that quercetin exerts a desirable toxic effect on GCSCs, and inhibits their survival. Although the exact mechanisms responsible for the quercetin-induced apoptosis of GCSCs remain unclear, some similarities should exist due to the homology between GCSCs and GCs (13). In 2012, it was hypothesized that quercetin-induced GC cell apoptosis was mediated via the mitochondrial intrinsic apoptotic pathway, which was an important theory that involved the release of apoptotic signals (35). The 
A

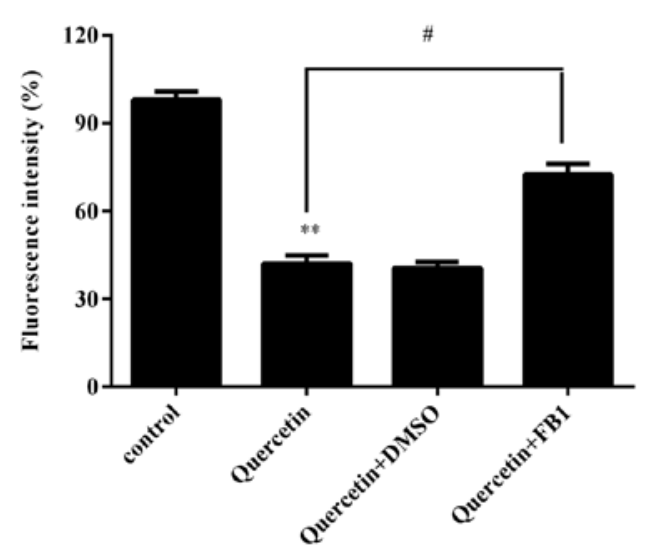

C

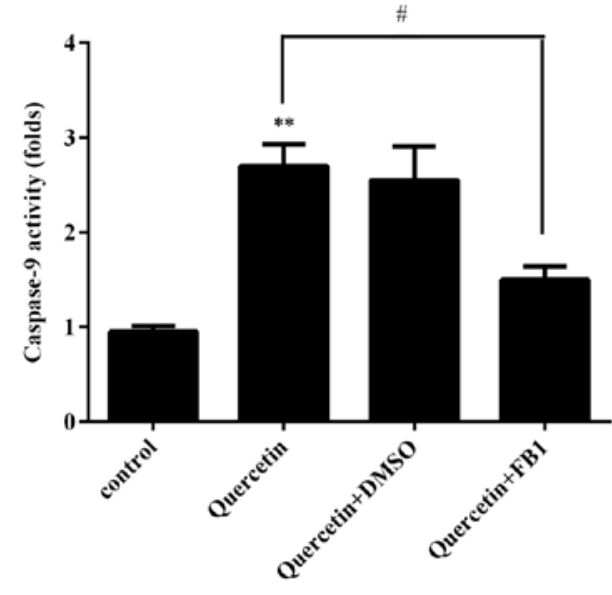

B

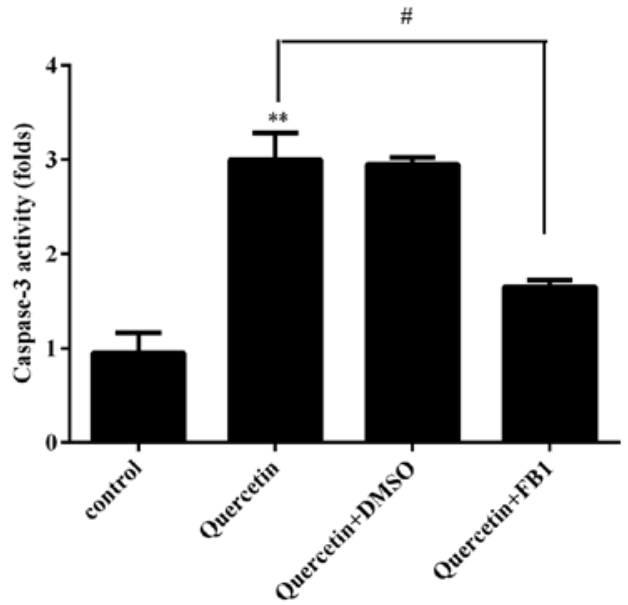

$\mathrm{D}$

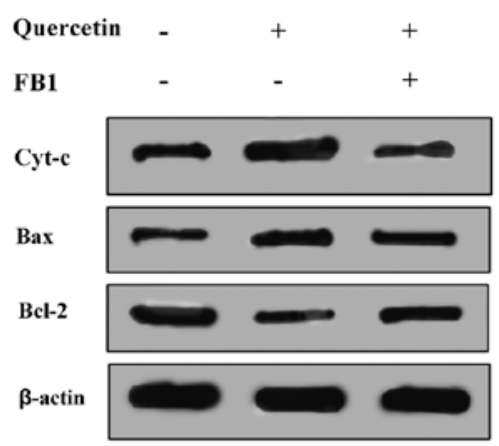

$\mathrm{E}$

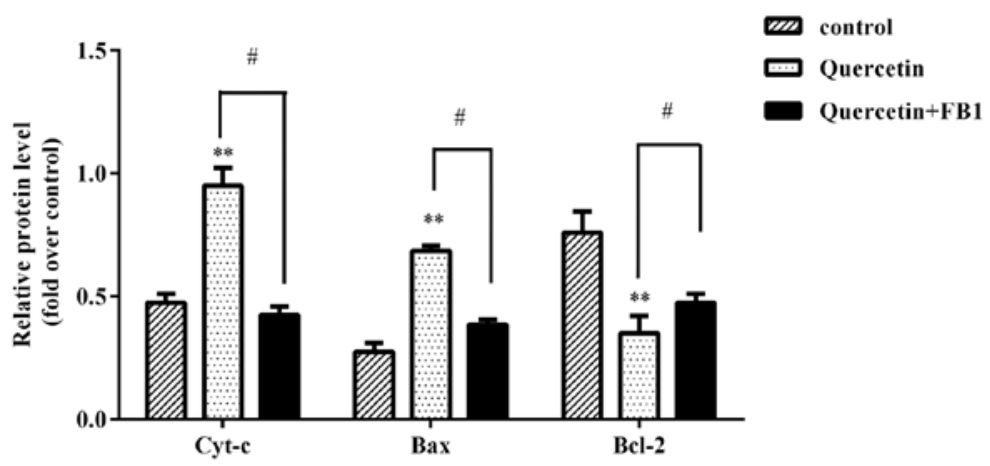

Figure 5. Effects of Akt activation on the sensitivity of apoptosis induced by quercetin. (A) Effects of fumonisin B1 (FB1, 25 $\mu \mathrm{M}$ ) on mitochondrial membrane potential. (B) Effects of FB1 on caspase-3. (C) Effects of FB1 on caspase-9. (D) Effects of FB1 on the expression of cytochrome $c$ (Cyt-c), Bax and Bcl-2. $\beta$-actin was used as an internal control. (E) The relative levels were normalized to those of $\beta$-actin. ${ }^{* *} \mathrm{p}<0.01$ vs. control group and ${ }^{\#} \mathrm{p}<0.05$.

mitochondrial apoptotic pathway in mammals is regulated by members of the Bcl-2 family of proteins (34). Similarly, in the present study, the collapse of the membrane potential, and the activation of caspase- 3 and caspase-9, as well as the upregulation of Cyt-c following treatment with quercetin, suggested the involvement of the mitochondrial apoptotic pathway. Moreover, the downregulation of the anti-apoptotic protein, $\mathrm{Bcl}-2$, and the upregulation of the pro-apoptotic protein, Bax, further reinforces the hypothesis that quercetin-induced growth inhibition occurrs through mitochondrial-dependent signaling in GCSCs.

$\mathrm{PI} 3 \mathrm{~K}-\mathrm{Akt}$ is a central regulatory protein which is involved in cell survival and plays a significant role in the regulation of many cellular functions, such as proliferation, cell viability and cell apoptosis $(27,28,36)$. In this study, we noted that Akt phosphorylation was decreased following treatment with quercetin. When the cells were pre-treated with the Akt activator, FB1, the quercetin-induced inhibition of the phosphorylation of Akt was markedly attenuated. Furthermore, the quecetininduced inhibition of cell growth and the enhancement of cell apoptosis were also attenuated by treatment with FB1. These data strongly suggest the involvement of the PI3K-Akt pathway in quercetin-induced growth inhibition, which is consistent with the findings of previous studies on the inhibitory effects of quercetin on Akt activation $(27,28,36)$. Based on previous 
studies which suggested that the PI3K-Akt pathway plays a negative role in the mitochondrial intrinsic pathway $(37,38)$, we confirmed the involvement of the PI3K-Akt pathway in the mitochondrial pathway. The effects of quercetin on the expression of Cyt-c, Bcl-2 and Bax, the activation of caspase-3 and caspase-9, as well as on mitochondrial membrane potential, were found to be significantly abated by FB1. These findings suggest that the inhibitory effecfts exerted by quercetin against GCSCs are regulated by the mitochondrial apoptotic pathway via the PI3K-Akt pathway.

In conclusion, in the present study, we provide evidence that quercetin has the potential to effectively intervene and prevent GCSC growth. Moreover, we noted that quercetin-induced apoptosis occurred via the mitochondrial-dependent pathway, which was mediated via the PI3K-Akt pathway. However, quercetin was less effective in the GCSCs than the MGC803 cells. Hence, promoting the specificity of quercetin to GCSCs is an aim for futuer research. We hypothesize that understanding the modulatory mechanisms of quercetin and promoting its specificity to GCSCs may aid in the development of quercetin as a potential chemotherapeutic agent for GC.

\section{References}

1. Boku N: HER2-positive gastric cancer. Gastric Cancer 17: 1-12, 2014.

2. Pang X, Wei W, Leng W, Chen Q, Xia H, Chen L and Li R: Radiotherapy for gastric cancer: a systematic review and metaanalysis. Tumour Biol 35: 387-396, 2014.

3. Jemal A, Center MM, DeSantis C and Ward EM: Global patterns of cancer incidence and mortality rates and trends. Cancer Epidemiol Biomarkers Prev 19:1893-1907, 2010.

4. Haraguchi N: ishii H, Sakai D, Satoh T, Doki Y and Mori M: The concept and significance of cancer stem cells. Nippon Geka Gakkai Zasshi 113: 204-209, 2012 (In Japanese).

5. Xu G, Shen J, Ou Yang X, Sasahara M and Su X: Cancer stem cells: the 'heartbeat' of gastric cancer. J Gastroenterol 48: 781-797, 2013.

6. Bonnet D and Dick JE: Human acute myeloid leukemia is organized as a hierarchy that originates from a primitive hematopoietic cell. Nat Med 3: 730-737, 1997.

7. Marsden CG, Wright MJ, Pochampally R and Rowan BG: Breast tumor-initiating cells isolated from patient core biopsies for study of hormone action. Methods Mol Biol 590: 363-375, 2009.

8. Collins AT, Berry PA, Hyde C, Stower MJ and Maitland NJ: Prospective identification of tumorigenic prostate cancer stem cells. Cancer Res 65: 10946-10951, 2005.

9. Yang ZF, Ngai P, Ho DW, Yu WC, Ng MN, Lau CK, Li ML, Tam KH, Lam CT, Poon RT and Fan ST: Identification of local and circulating cancer stem cells in human liver cancer. Hepatology 47: 919-928, 2008

10. O'Brien CA, Pollett A, Gallinger S and Dick JE: A human colon cancer cell capable of initiating tumour growth in immunodeficient mice. Nature 445: 106-110, 2007.

11. Li C, Lee CJ and Simeone DM: Identification of human pancreatic cancer stem cells. Methods Mol Biol 568: 161-173, 2009.

12. Dewi DL, Ishii H, Kano Y, Nishikawa S, Haraguchi N, Sakai D, Satoh T, Doki Y and Mori M: Cancer stem cell theory in gastrointestinal malignancies: recent progress and upcoming challenges. J Gastroenterol 46: 1145-1157, 2011.

13. Li K, Dan Z and Nie YQ: Gastric cancer stem cells in gastric carcinogenesis, progression, prevention and treatment. World J Gastroenterol 20: 5420-5426, 2014.

14. Zhou ZH, Zhang JD, Xu GF and Zhao HB: Isolation of gastric cancer stem cells based on colony morphology and detection of their sensitivity to 5-fluorouracil. Zhonghua Wei Chang Wai Ke Za Zhi 16: 376-380, 2013 (In Chinese).

15. Liu J, Ma L, Xu J, Liu C, Zhang J, Liu J, Chen R and Zhou Y: Spheroid body-forming cells in the human gastric cancer cell line MKN-45 possess cancer stem cell properties. Int J Oncol 42: 453-459, 2013.

16. Boots AW, Haenen GR and Bast A: Health effects of quercetin: from antioxidant to nutraceutical. Eur J Pharmacol 585: 325-337, 2008 .
17. Russo GL, Russo M, Spagnuolo C, Tedesco I, Bilotto S, Iannitti R and Palumbo R: Quercetin: a pleiotropic kinase inhibitor against cancer. Cancer Treat Res 159: 185-205, 2014.

18. Wang K, Liu R, Li J, Mao J, Lei Y, Wu J, Zeng J, Zhang T, Wu H, Chen L, et al: Quercetin induces protective autophagy in gastric cancer cells: Involvement of Akt-mTOR- and hypoxia-induced factor 1 $\alpha$-mediated signaling. Autophagy 7: 966-978, 2011.

19. Wang HY, Guo LM, Chen Y, Zhao XH, Cheng CL, Wu MY and He LY: Quercetin inhibits growth and induces apoptosis of human gastric carcinoma cells. Xi Bao Yu Fen Zi Mian Yi Xue Za Zhi 22: 585-587, 2006 (In Chinese).

20. Yoshida M, Sakai T, Hosokawa N, Marui N, Matsumoto K, Fujioka A, Nishino $\mathrm{H}$ and Aoike A: The effect of quercetin on cell cycle progression and growth of human gastric cancer cells. FEBS Lett 260: 10-13, 1990.

21. Adikrisna R, Tanaka S, Muramatsu S, Aihara A, Ban D, Ochiai T, Irie T, Kudo A, Nakamura N, Yamaoka S and Arii S: Identification of pancreatic cancer stem cells and selective toxicity of chemotherapeutic agents. Gastroenterology 143: 234-245 e237, 2012.

22. Srivastava RK, Tang SN, Zhu W, Meeker D and Shankar S: Sulforaphane synergizes with quercetin to inhibit self-renewal capacity of pancreatic cancer stem cells. Front Biosci (Elite Ed) 3 : 515-528, 2011.

23. Zhou W, Kallifatidis G, Baumann B, Rausch V, Mattern J, Gladkich J, Giese N, Moldenhauer G, Wirth T, Büchler MW, et al: Dietary polyphenol quercetin targets pancreatic cancer stem cells. Int J Oncol 37: 551-561, 2010.

24. Tang SN, Singh C, Nall D, Meeker D, Shankar S and Srivastava RK: The dietary bioflavonoid quercetin synergizes with epigallocathechin gallate (EGCG) to inhibit prostate cancer stem cell characteristics, invasion, migration and epithelial-mesenchymal transition. J Mol Signal 5: 14, 2010.

25. Chang WW, Hu FW, Yu CC, Wang HH, Feng HP, Lan C, Tsai LL and Chang YC: Quercetin in elimination of tumor initiating stem-like and mesenchymal transformation property in head and neck cancer. Head Neck 35: 413-419, 2013.

26. Li C, Heidt DG, Dalerba P, Burant CF, Zhang L, Adsay V, Wicha M, Clarke MF and Simeone DM: Identification of pancreatic cancer stem cells. Cancer Res 67: 1030-1037, 2007.

27. Granado-Serrano AB, Martín MA, Bravo L, Goya L and Ramos S: Quercetin induces apoptosis via caspase activation, regulation of Bcl-2, and inhibition of PI-3-kinase/Akt and ERK pathways in a human hepatoma cell line (HepG2). J Nutr 136: 2715-2721, 2006

28. Xiang T, Fang Y and Wang SX: Quercetin suppresses HeLa cells by blocking PI3K/Akt pathway. J Huazhong Univ Sci Technolog Med Sci 34: 740-744, 2014.

29. Pan HC, Jiang Q, Yu Y, Mei JP, Cui YK and Zhao WJ: Quercetin promotes cell apoptosis and inhibits the expression of MMP-9 and fibronectin via the AKT and ERK signalling pathways in human glioma cells. Neurochem Int 80: 60-71, 2015.

30. Takaishi S, Okumura T and Wang TC: Gastric cancer stem cells. J Clin Oncol 26: 2876-2882, 2008

31. Stojnev S, Krstic M, Ristic-Petrovic A, Stefanovic V and Hattori T: Gastric cancer stem cells: therapeutic targets. Gastric Cancer 17: 13-25, 2014.

32. Liu J, Ma L, Wang Z, Wang L, Liu C, Chen R and Zhang J: MicroRNA expression profile of gastric cancer stem cells in the MKN-45 cancer cell line. Acta Biochim Biophys Sin (Shanghai) 46: 92-99, 2014

33. Borska S, Chmielewska M, Wysocka T, Drag-Zalesinska M, Zabel $\mathrm{M}$ and Dziegiel P: In vitro effect of quercetin on human gastric carcinoma: targeting cancer cells death and MDR. Food Chem Toxicol 50: 3375-3383, 2012.

34. Brunelle JK and Letai A: Control of mitochondrial apoptosis by the Bcl-2 family. J Cell Sci 122: 437-441, 2009.

35. Wang $\mathrm{P}$, Zhang K, Zhang Q, Mei J, Chen CJ, Feng ZZ and Yu DH: Effects of quercetin on the apoptosis of the human gastric carcinoma cells. Toxicol In Vitro 26: 221-228, 2012.

36. Spencer JP, Rice-Evans C and Williams RJ: Modulation of prosurvival Akt/protein kinase B and ERK1/2 signaling cascades by quercetin and its in vivo metabolites underlie their action on neuronal viability. J Biol Chem 278: 34783-34793, 2003

37. Zhang Y, Sun S, Chen J, Ren P, Hu Y, Cao Z, Sun H and Ding Y: Oxymatrine induces mitochondria dependent apoptosis in human osteosarcoma MNNG/HOS cells through inhibition of PI3K/Akt pathway. Tumour Biol 35: 1619-1625, 2014.

38. Cheong JW, Min YH, Eom JI, Kim SJ, Jeung HK and Kim JS: Inhibition of CK $2 \alpha$ and PI3K/Akt synergistically induces apoptosis of $\mathrm{CD}^{+} 4^{+} \mathrm{CD} 38^{-}$leukaemia cells while sparing haematopoietic stem cells. Anticancer Res 30: 4625-4634, 2010. 